مطالعه همبستكى و تجزيه عليت صفات زراعى و عملكرد دانه جمعيتهاى كَندم نياى

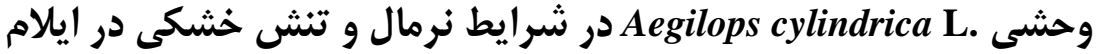

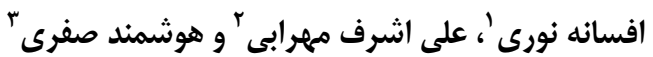

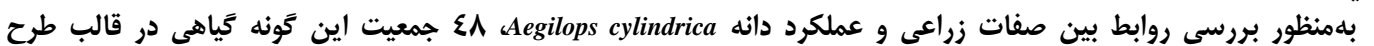

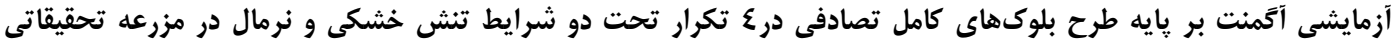

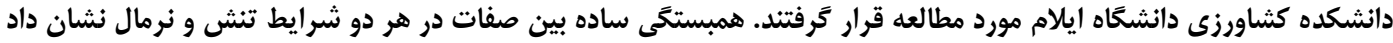

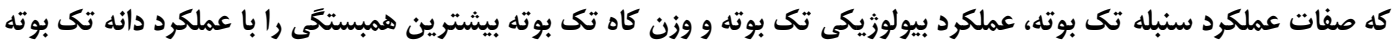

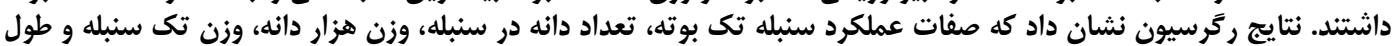

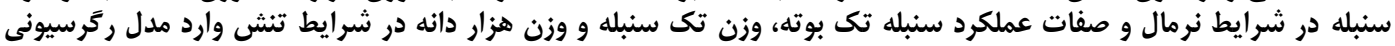

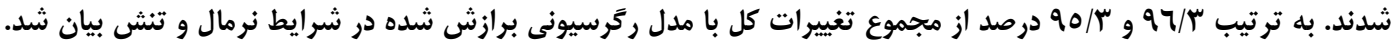

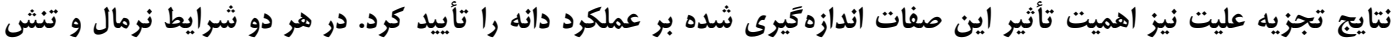

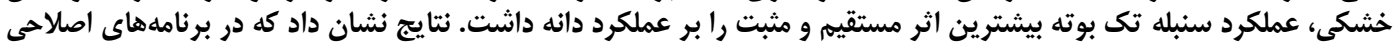

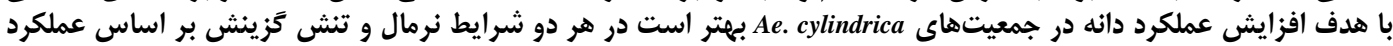

سنبله صورت يذيرد.

وازههاى كليدى: آزيلويس سيلندريكا، تجزيه همبستكى، تجزيه عليت، تنش خشكى، عملكرد دانه

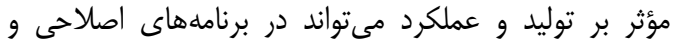

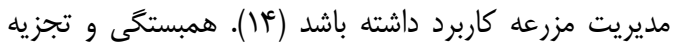

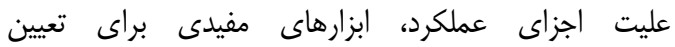

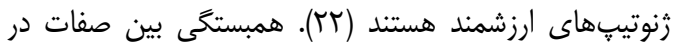

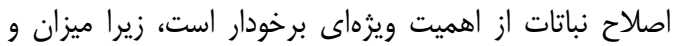

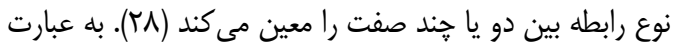

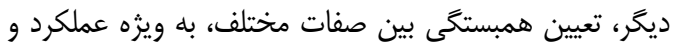

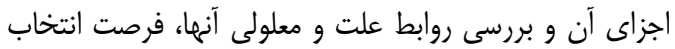

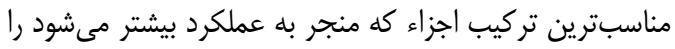

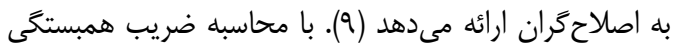

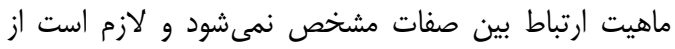

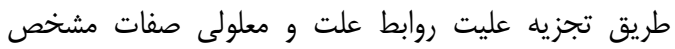

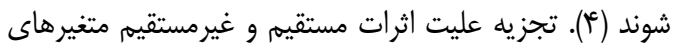

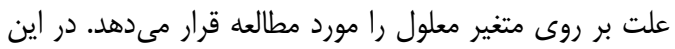

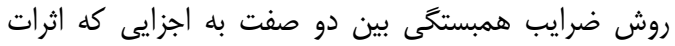

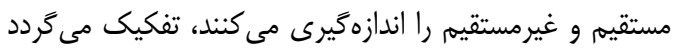
(r)

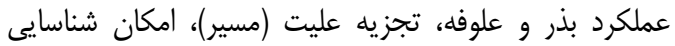

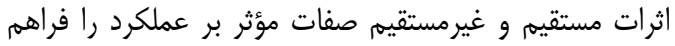

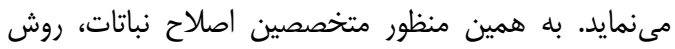

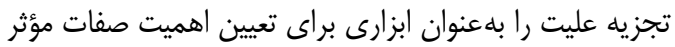

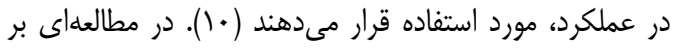

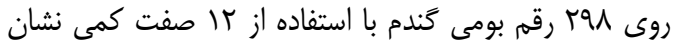

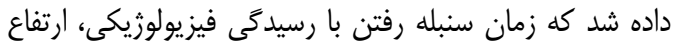

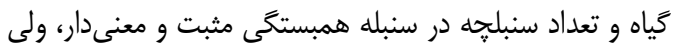

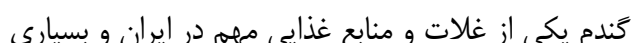

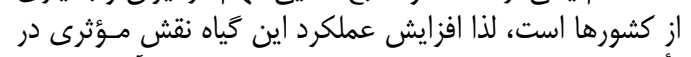

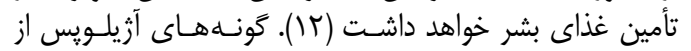

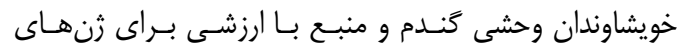

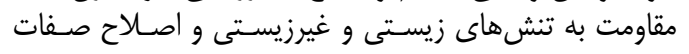

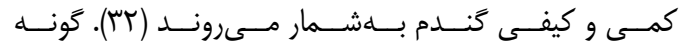
Aegilops cylindrica

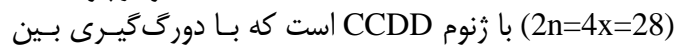
كونهاى دييلوئيد 2x=14)Ae. tauschii 2n=2x=14) Ae. caudate

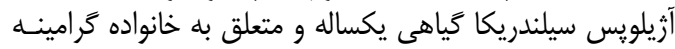

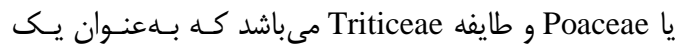

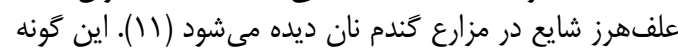

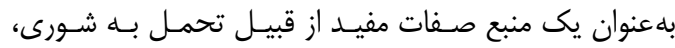

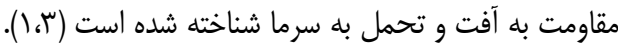

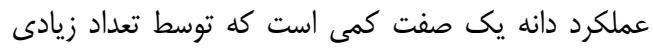

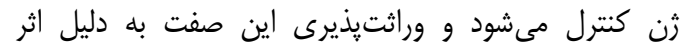

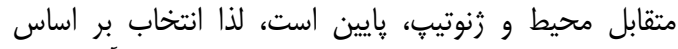

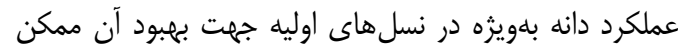

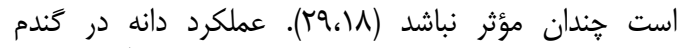

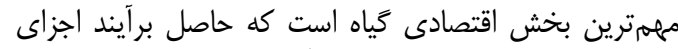

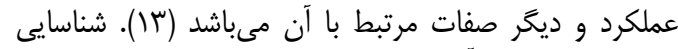

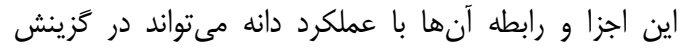
واريتههاى يرمحصول مؤثر واقع شود. همجنين شناخت ران صفات 
تحقيق حاضر بلمنظور تعيين همبستخى و مشخص نمودن

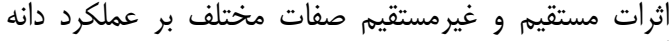
آزيلويس سيلندريكا انجام گرفته است.

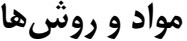

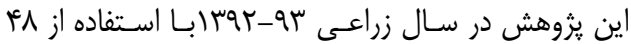

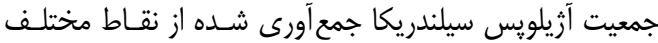

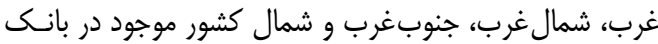

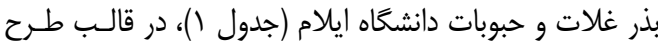

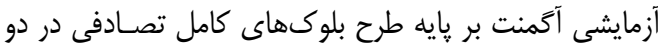

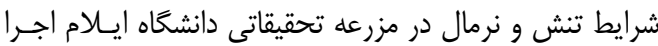

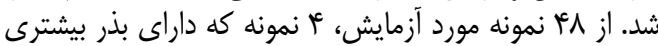

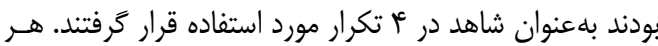

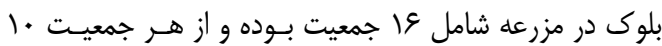

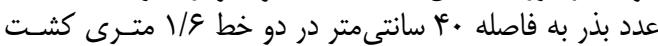

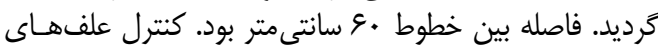

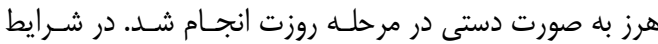

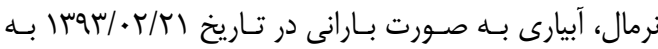

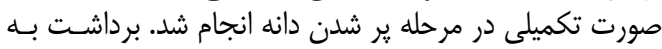

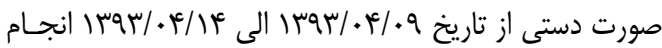

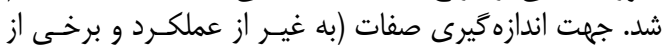

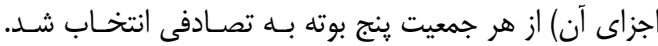

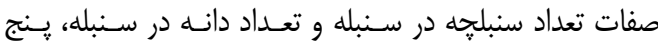

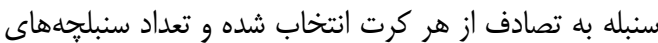

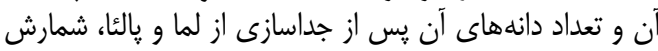

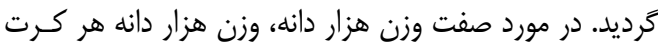

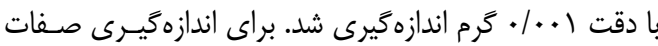

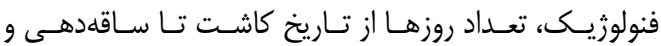

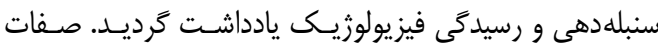

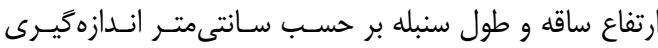

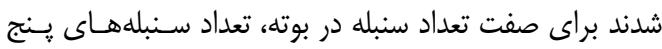

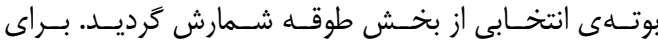

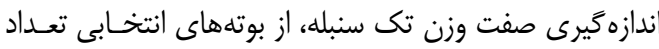

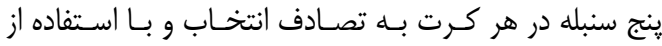

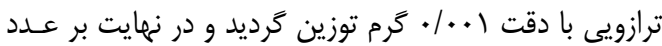
ينج تقسيم شد.

براى اندازهيرى عملكرد سنبله تكى بوته و و عملكرد

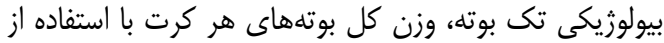

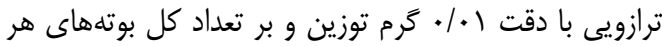

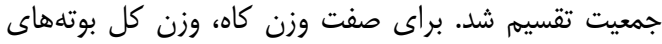

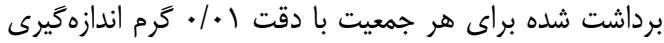

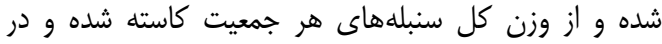

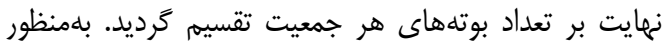

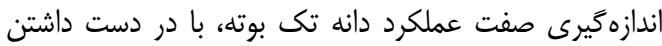

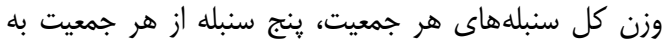

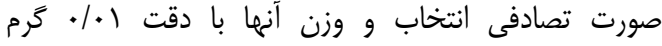

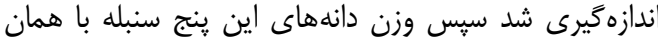

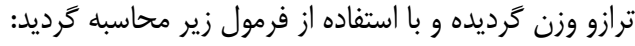

با وزن هزار دانه، عملكرد بيولوزيكى و عملكرد دانه همبستكى إنى

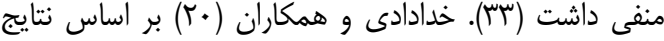

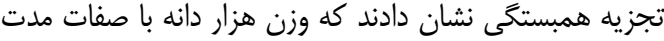

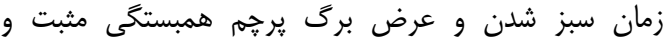

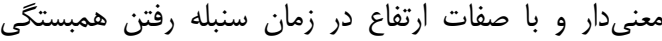
منفى و معنى دار داشت. در مطالعهى هانى

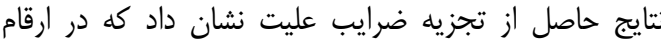

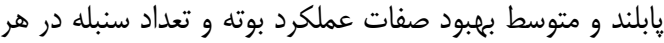

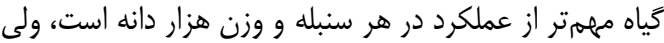

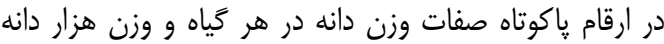

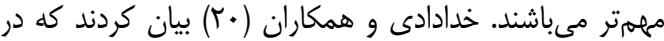

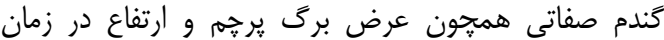

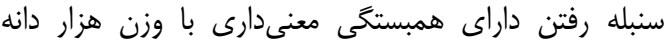
بودهاند و به همراه صفات طول سنبله بdطور معنىدارى تغييرات وزن هزار دانه رات رات توجيه نمودند.

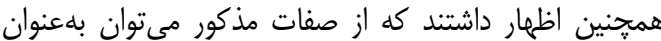

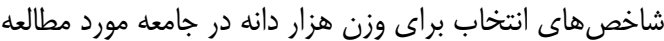

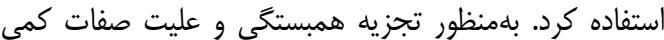

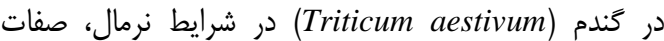

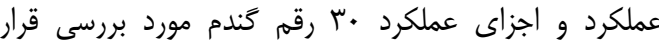

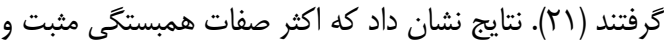
معنى دارى در هر دو سطح فنوتيبى و زنوتيبى داشتند. همجنين

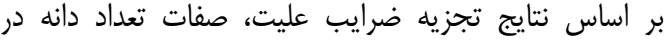

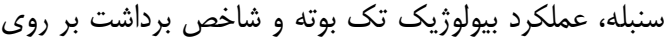

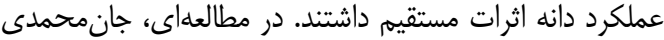

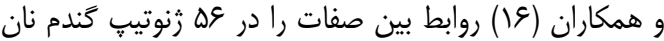
در شرايط مزرعه بررسى كردند. نتايج تجزيه روات همبستخى نشات نشان

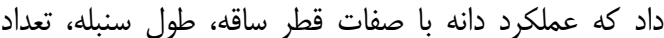

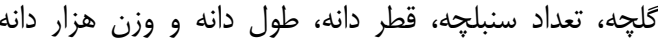

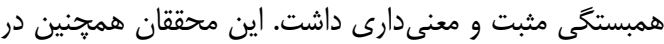

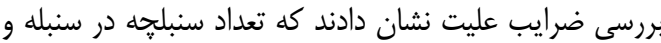

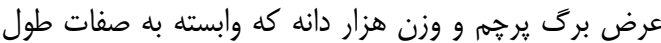

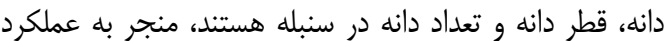

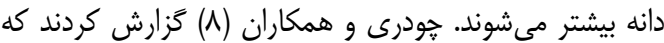

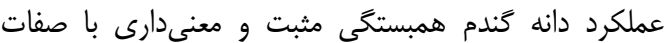

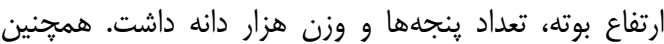

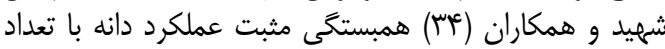

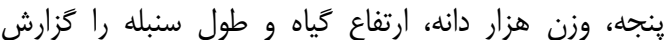

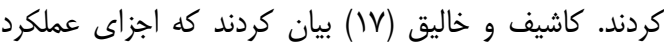
دانه شامل تعداد سنبله در بوته، تعداد دانه در دان سنبله

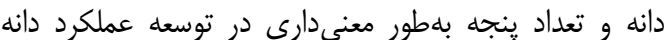

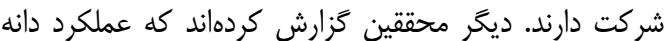

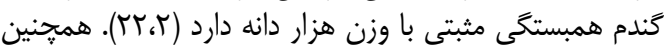

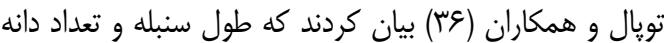

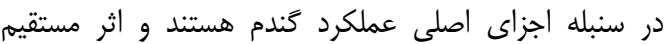

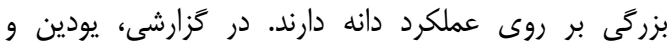

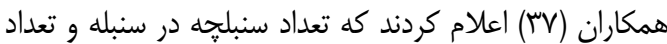
ينجه بيشترين اثر مستقيم را بر عملكرد دانه دانه دارند. 
بهنوان متغيرهاى مستقل مورد تجزيه و تحليل قرار گرفتند.

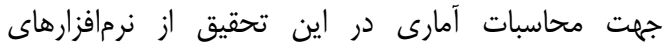
SAS 9.0
وزن دانههاى ×وزن كل سنبلهها) = عملكرد دانه تكى بوته

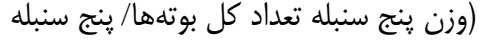

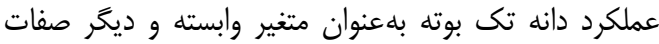

جدول ا- مشخصات و محل جمع آورى جمعيتهاى Ae. cylindrica مورد استفاده در اين تحقيق

Table 1. Characterization and origin of Ae. cylindrica populations which used in this research

\begin{tabular}{|c|c|c|c|c|c|}
\hline محل جمع آورى & كد بانك بذر & كد مورد استفاده در & محل جمع آورى & كد بانك بذر & آزتمادماده درد \\
\hline زنجان - ورودى جاده زنجان - تهران & IUGB-00239 & TQ & كيلوسترى درود جاده اليخودرز-درود ، لر & IUGB-00210 & 1 \\
\hline كرمانشاه- هرسين - نزديك روستاى خغاسعيد & IUGB-00090 & te & 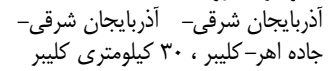 & IUGB-00202 & $r$ \\
\hline كرمانشاه- سنقر - اسدآباد- روستاى ياسر & IUGB-00200 & tr & كردستان-دهگلان & IUGB-02073 & r \\
\hline اردبيل - جاده اردبيل - مشخين شهر & IUGB-00150 & ru & كرمانشاه- دالاهو - كرند & IUGB-01520 & f \\
\hline 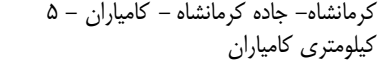 & IUGB-00097 & ra & اردبيل - جاده اردبيل- مشخين شهر & IUGB-00271 & $\Delta$ \\
\hline تبريز آذربانان شرقى - • اكيلومترى جاده اهر - & IUGB-00201 & r. & شوراب - جاده خرم آباد - انديمشك - & IUGB-00059 & 8 \\
\hline آذربايجان شرقى - • كيلومترى اهر - كليبر & IUGB-00156 & 衤 & كبال بختيارى - جاده ناغان- ايذه & IUGB-00236 & v \\
\hline كرمانشاه- جاده هرسين - كرمانشاه - كرسين & IUGB-00132 & m & 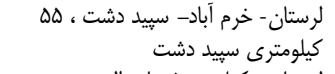 & IUGB-00391 & $\wedge$ \\
\hline كرمانشاه- - جاده سنقر & IUGB-00248 & سז & فيروزآباد - كيلومتر ه جاده الشتر- & IUGB-00229 & 9 \\
\hline كرمانشاه- جاده اسدآباد - همدان & IUGB-00388 & mF & لرستان- درود - ترش آب & IUGB-01359 & $1 \cdot$ \\
\hline كرمانشاه- سلام آباد - سرمست & IUGB-01598 & ra & - روستاى حيران & IUGB-00258 & 11 \\
\hline لرستان- • سكيلومترى خرم آباد - سييد دشت & IUGB-00189 & 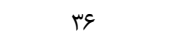 & كان شرقى- كيلومتر •ع اهر - & IUGB-00188 & it \\
\hline كرمانشاه- هرسين & IUGB-00034 & rv & كرمانشاه- هرسين & IUGB-00376 & س \\
\hline جهارمهال و بختيارى - حومه شهركرد & IUGB-00373 & щ人 & لرستان- بروجرد - همت آباد & IUGB-01238 & $1 f$ \\
\hline كرمانشاه-دو راهى جوانرود . & IUGB-00417 & rq & & IUGB-01208 & 10 \\
\hline لرستان - نورآباد - روستاى ده سفيد & IUGB-00062 & f. & - مسير هرسين - نورآباد & IUGB-00078 & 19 \\
\hline كرمانشاه- بعد از زرينه به سمت سقز & IUGB-00359 & (1) & 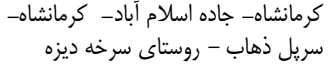 & IUGB-00095 & IV \\
\hline كيلان-رشت & IUGB-00153 & ft & & IUGB-01592 & $M$ \\
\hline جاده اردبيل - سرعين ، ها كيلومترى سرعين & IUGB-00221 & et & - جاده سومعه سرا - آستارا & IUGB-00185 & 19 \\
\hline كرمانشاه- - جاده هرسين كرمانشاه روستاى & IUGB-00168 & if & ورودى جاده زنجان - تهران & IUGB-00172 & $r \cdot$ \\
\hline كرمانشاه- سريل ذهاب - ريزه وند - نجف & IUGB-01213 & $F \Delta$ & تمبان - •ه كيلومترى جاده زنجان - & IUGB-00390 & rl \\
\hline شاويار مانشاه- جاده جوانرود - ياوه - روستاى & IUGB-00065 & is & زيرتل كهيلويه و بوير احمد- ياسوج - محله & IUGB-00403 & tr \\
\hline 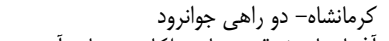 & IUGB-00406 & fr & كَيلان - جاده فومن - تالش & IUGB-00270 & זr \\
\hline آذربايجان شرقى- جاده ملكان - مياندوآب & IUGB-00241 & is & كيلان - جاده رشت - فومن & IUGB-00267 & te \\
\hline
\end{tabular}

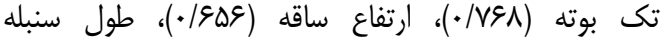

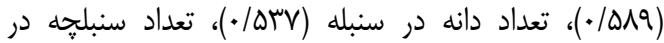

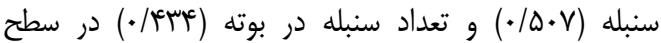

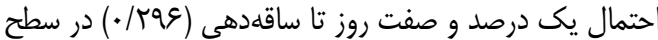

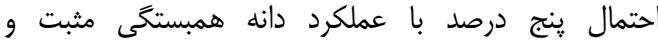

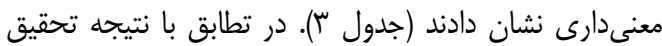

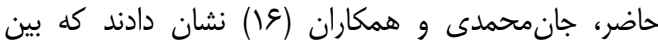

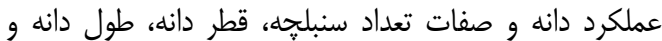

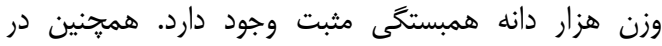

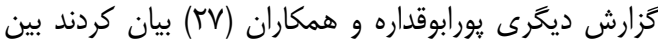

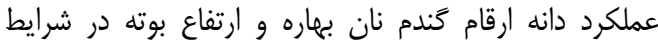

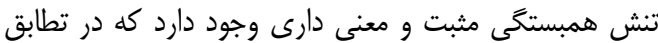
با نتايج بدست آمده از شرايط تنش در در اين يثروهش است.
نتايج و بحث

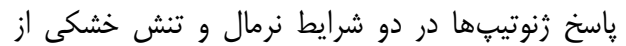

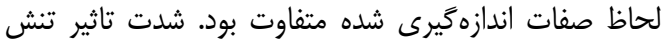

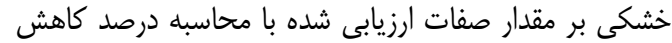

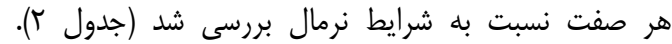

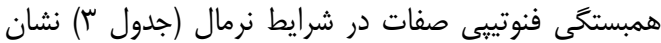

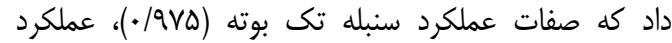

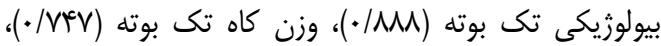

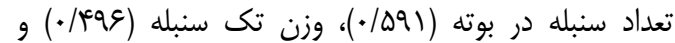

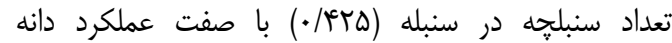

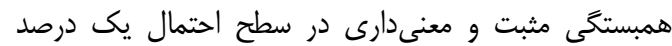

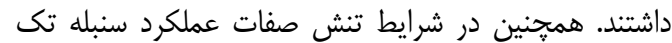

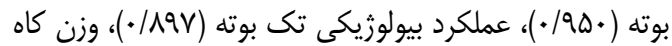


Ae. cylindrica جدول r- درصد تغييرات ناشى از تنش خشكى بر صفات مورد مطالعه دالعiات Table 2. Percentage of variation due to drought stress on studied traits in Ae. Cylindrical

\begin{tabular}{|c|c|c|c|c|}
\hline درصد تغيير صفات & ميزان تغيير صفات & ميانكين صفات در & شرايطئن صفات در & صفات \\
\hline$r / 4 \varphi^{2}$ & $\cdot / \pi \Lambda^{\mathrm{nS}}$ & $V / q 9 \pm \cdot / q)$ & $N / T \Delta \pm \cdot / 9 T$ & تعداد سنبلحه در سنبله \\
\hline$V / 99$ & $1 / 11^{*}$ & $\mid r / r y \pm r / .9$ & $\mid F / F F \pm 1 / q D$ & تعداد دانه در سنبله \\
\hline r/ r & $\cdot / \cdot \Delta^{\mathrm{ns}}$ & س & $\mid / F I \pm \cdot / r \Delta$ & وزن ... إنه (g) \\
\hline$-1 / v$ & $-r / / f^{n+\infty}$ & $|A V / r \cdot \pm F / V|$ & $|A F / \cdot \varepsilon \pm \varepsilon| \cdot 1$ & روز تا ساقدهى \\
\hline$-1 / 1 \Lambda$ & $-T / M F^{*}$ & $19 r / F V \pm F / 9 \Delta$ & $19 \cdot / \kappa+ \pm \Delta / \kappa \varepsilon$ & روز تا سنبلهدهى \\
\hline.$/ 41$ &.$/ 90^{\mathrm{ns}}$ & $r \mu / \Delta S \pm \Delta / q$. & $T \mu / \Delta / \pm \Delta / T^{\prime}$ & روز تا •هـ٪ رسيدىى فيزيولوزيك \\
\hline$V / \Delta S$ & $r / v^{n-r}$ & $F \Delta / r F \pm \varepsilon / \Delta q$ & $\kappa q / \cdot \Delta \pm \psi / \cdot \psi^{c}$ & ارتفاع ساقه (cm) \\
\hline$-r / 9)$ & $-\cdot / \kappa \Delta^{n s}$ & $\mid Q / \Lambda \vee \pm 1 / \Delta \Lambda$ & $\mid Q / F T \pm 1 / M$ & طول سنبله (cm) \\
\hline 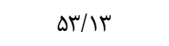 & $r \varepsilon / . q^{r n}$ & $r / \cdot r \pm I D / F \Delta$ & $|r q / \| \pm \pm r| / r$. & تعداد سنبله در بوته \\
\hline$\Delta \cdot / 9 \mu$ & $N / r^{* N}$ & V/११ะF//Q & $\mid \varepsilon / 19 \pm \varepsilon / \Delta V$ & عملكرد سنبله تك بوته (g) \\
\hline$\Delta S / \Delta S$ & $r \cdot / \Lambda f^{n-T}$ & $\mid \varepsilon / \cdot 1 \pm 9 / 1 V$ & r $/ \wedge \Delta \pm \mid V / 11$ & عملكرد بيولوزيكى تك بوته (g) \\
\hline$\Delta s / q 1$ & $F / T F^{n r}$ & $r / r \mid \pm 1 / 9 \Delta$ & $V / F \Delta \pm r / \lambda)$ & عملكرد دانه تك بوته (g) (0) \\
\hline (91/ז1 & $\mid r / 9 q^{n+}$ & $V / 9 \Lambda \pm \Delta / 19$ & $r \cdot / 9 V \pm I I / \& r$ & وزن كاه تك بوته (g) \\
\hline
\end{tabular}

جدول ب- ضرايب همبستكى ساده براى صفات ارزيابى شده روى جمعيتهاى آزيلويس سيلندريكا در شرايط تنش خشكى (يايين قطر) و شرايط نرمال (بالاى قطر)

Table 3. Correlation coefficients for evaluated traits on Ae. cylindrica populations under drought stress conditions (below diameter) and normal conditions (above diameter)

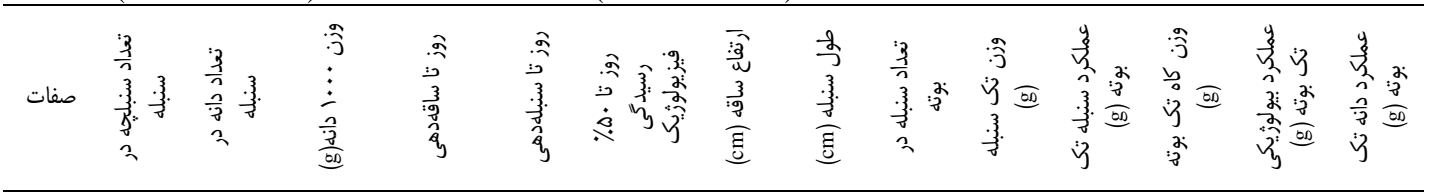

\begin{tabular}{|c|c|c|c|c|c|c|c|c|c|c|c|c|c|c|}
\hline & SPS & $\mathrm{GpS}$ & HGNo & DtSh & DtH & DtM & ShootL & SpikeL & EpP & SSW & SpikeY & StrawW & Biomass & GrainY \\
\hline SPS & 1 & . /994 &.$- / . r$. & $\cdot / \mu \Delta \Delta^{*}$ & . & 酸. &.$/ \mu V \cdot{ }^{* \pi}$ & $.109)^{-\pi}$ &.$/ r V r^{n \pi}$ &.$|F \Delta|^{\prime \prime m}$ & $\cdot / f \cdot f^{* m}$ & $\cdot / F M^{* *}$ & 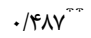 & . / FTO \\
\hline $\mathrm{GpS}$ & $\cdot / A V V^{*-\sigma}$ & 1 & $-\cdot / T V^{c}$ & . & $\cdot / F \Delta F^{N T}$ & 西/ & $\cdot / \mu \Delta \cdot{ }^{*}$ & . & $\cdot / T \Delta q$ & . & . MaY & $\cdot / r V g^{* * *}$ & $\cdot / \mu / \Delta^{*}$ & $\cdot / T \Delta$. \\
\hline HGNo & $-0 /$ RTE & - & 1 & - &.$- / 49 v$ &.$- / 419$ &.$- / . r v$ & $-\cdot|| Q \mid$ & $\cdot / 1 \cdot r$ &.$/ N F$ &.$/ 194$ & $. / 14 \mid$ & $\cdot|| V \mid$ & $\cdot / r \cdot 1$ \\
\hline DtSh & $\cdot /$ Frq $^{* \pi}$ & $\cdot / \Delta \cdot . \cdot$ & $-\cdot / 4 \mid q^{n-m}$ & 1 & $\cdot / v \Lambda \cdot{ }^{* m}$ & $\cdot / \Gamma \Delta T^{*}$ & "אזץ/א. & - $/ \Delta F \Delta^{* m}$ & $.1 \cdot 19$ &.$- / \cdot 1$ &.$- / 114$ &.$/ \ldots$ & . .4 . & $-11 \cdot{ }^{f}$ \\
\hline DtH & $\cdot \mid \Delta \Delta \Psi^{n-T}$ &.$/ \Delta 9 F^{-m}$ & $-\cdot / \mathrm{rq}^{-* *}$ &.$/ A \& S^{n-m}$ & 1 & $. / F \mid f^{-m}$ & "T & $\cdot / \Delta \Lambda \cdot{ }^{m *}$ & $\cdot \pi \cdot \Delta$ &.$/ .1 \mathrm{~V}$ & $\cdot / \cdot \wedge \mathrm{V}$ & $\cdot / T \cdot \Delta$ &.$/|V|$ & $\cdot / \cdot V^{c}$ \\
\hline DtM & $\cdot / r \cdot V$ &.$/ r \Delta$. &.$- / 111$ &.$/ F v \mu^{n *}$ & . $/ \Delta \Delta \mu^{n \uparrow}$ & 1 & -.1 .19 &.$/ \% 99^{\circ}$ &.$- / 19$. & $\% \Delta$ & .1 .48 &.$- / I V W$ & -.1 .99 & $.1 . \times 9$ \\
\hline ShootL & $\cdot / N T D^{-m}$ & $\cdot / V \in V^{n-m}$ &.$- / .+v V$ & $\cdot / \Delta \cdot \Delta^{N \pi}$ & $\cdot / \Delta M^{\pi \omega}$ & $\cdot 1 \cdot v 1$ & 1 &.$/ F V V^{m \omega}$ & $\cdot / \backslash Q \Lambda$ & . /IQT & . & . & $. / F \mid f^{n-m}$ & هזו/. \\
\hline SpikeL & $\cdot / V \mid{ }^{n-m}$ & $\cdot \mid \& M^{\pi *}$ & $-.11 \cdot 9$ & $\cdot \mid \Delta \Delta \Phi^{n-T}$ & $\cdot / \Delta V f^{n-T}$ & $\cdot / \Gamma V \Lambda^{m *}$ & . $/$ YY & 1 &.$/ r 9 \Lambda^{\circ}$ & . &.$/ \% 90^{\circ}$ & 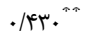 & $\cdot / F \cdot \varphi^{n-m}$ & . \\
\hline EpP & $\cdot|\Delta f|^{-\pi}$ & . & $-. \cdot / \cdot \cdot 1$ & $\cdot / \Delta \cdot \Lambda^{\pi T}$ & $\cdot / \Delta F \cdot{ }^{m T}$ & •/TVG &.$/ 999^{\prime \prime \prime}$ & 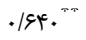 & 1 & 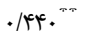 &.$/ 0 \wedge 9^{* \pi}$ & 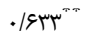 & $\cdot \mid \& \Delta V^{\prime \prime m}$ & $.109)^{n-m}$ \\
\hline SSW & $\cdot / F V \varepsilon^{n \pi}$ & •/ & .1 .4$. & .1 .94 &.$/ 11$. & .1 .9$. &.$/ T M{ }^{\circ}$ & 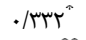 & . TAK & 1 &.$|\Delta| \varepsilon^{\pi m}$ &.$/ F I V^{n-m}$ & $\cdot / \propto \wedge \Delta D^{* \pi}$ &.$/ 499^{* \pi}$ \\
\hline SpikeY &.$/ 819^{\circ \pi}$ & $\cdot / \Delta Q \varphi^{n *}$ & $\cdot|\cdot v|$ & . & $\cdot / T V$ & .1 .11 & . $/$ NTq & $\cdot|q V|^{n-m}$ & $\cdot|\Delta| 9^{N T}$ & "זسא/. & 1 & 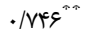 & $\cdot / 19 \Lambda^{\circ \pi}$ & . $/ q \vee \Delta^{* \pi}$ \\
\hline StrawW & $\cdot / \Delta \Delta \Lambda^{\circ m}$ & . $/ \Delta \& S^{*-F}$ & $-\cdot / \cdot v 1$ & •/\%q & $\cdot / r W w^{* *}$ &.$- / \cdot V r$ & •/Al|r" & $\cdot \mid q \cdot f^{* m}$ &.$/ 990^{N \pi}$ & $\cdot / r \cdot 9$ & $\cdot / A T \Lambda^{* T}$ & 1 & . $/ 99 \mu^{* T}$ & $\cdot / V \in V^{* \pi}$ \\
\hline Biomass & $\left.\cdot|9|\right|^{* \pi}$ & $\cdot / 9 \cdot r^{* \tau}$ &.$- / .+\varphi^{c}$ & . & . & $-.1 .4 \Lambda$ & $\cdot / \Lambda \cdot V^{* n}$ & $.199 T^{* \pi}$ &.$|91|^{.17}$ & $\cdot / 4 \cdot \varphi^{\pi}$ &.$/ 909^{* \pi}$ & $. / 94)^{n-\pi}$ & 1 & $\cdot / M^{*}$ \\
\hline GrainY & $\cdot / \Delta \cdot V^{-\pi}$ & $\cdot / \Delta \Psi v^{N T}$ & / MFF & . & צמא/. & $.1 . .9$ &. $\mid \angle \Delta S^{\prime \prime N}$ & $\cdot 1019^{m \pi}$ &.$/ F M f^{N T}$ & .11 .9 & $\cdot /$ $_{\Delta} \cdot{ }^{* \pi}$ & $\cdot /$ VYN & $\cdot / \wedge q \gamma^{* \sigma}$ & 1 \\
\hline
\end{tabular}

و زنوتييى بيان داشتند. در مطالعه حاضر تعداد سنبلجه در

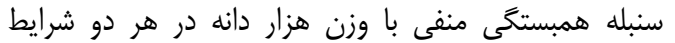

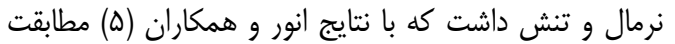

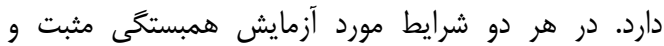

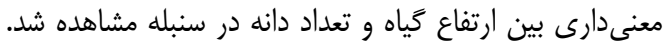

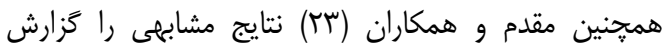

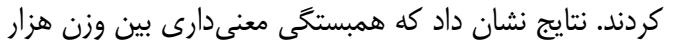

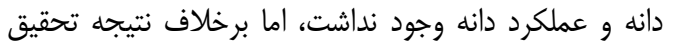

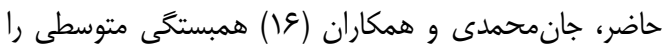

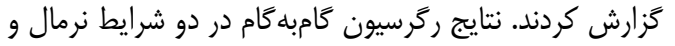

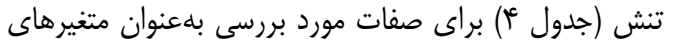

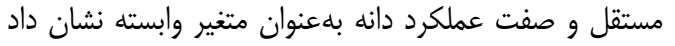

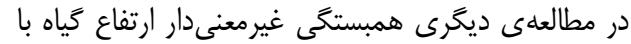

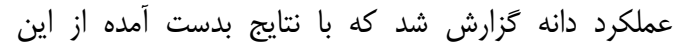

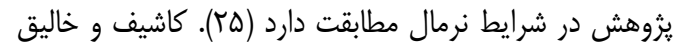

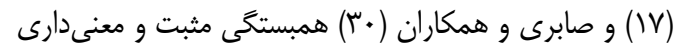

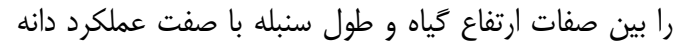

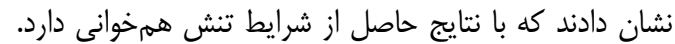

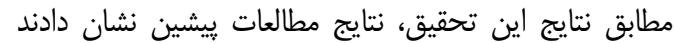

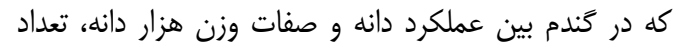

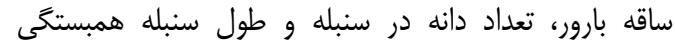

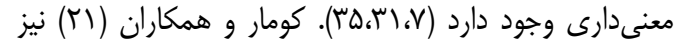

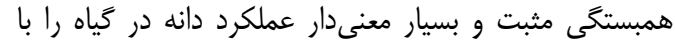
تعداد دانه در گياه و شاخص برداشت در هر در دو سطح فنوتييى 


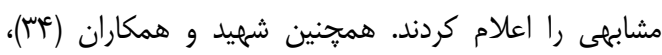

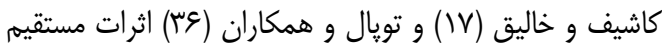

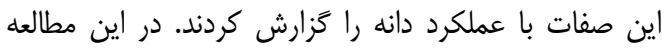

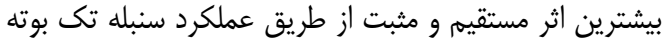

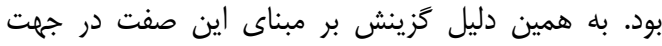

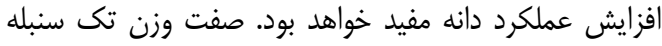

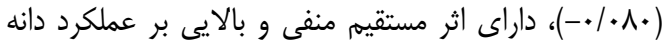

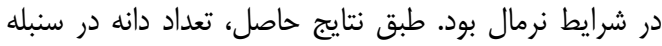

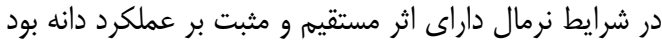

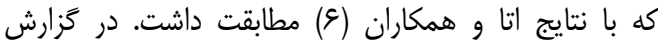

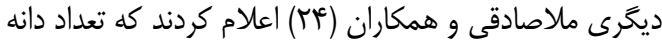

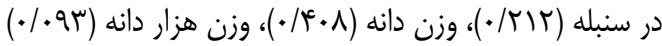

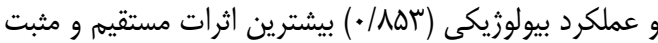

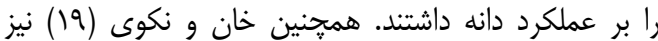

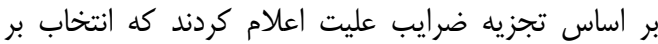

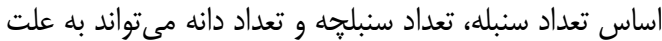

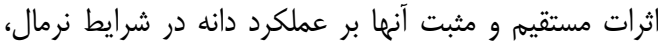

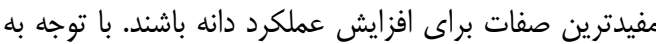

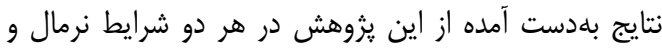

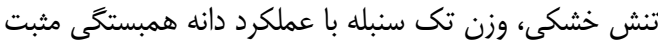

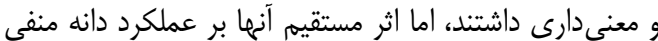

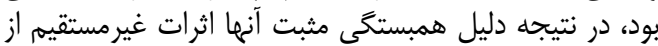

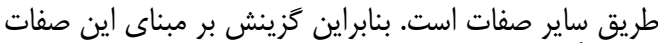

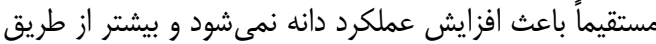

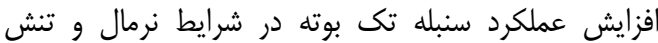

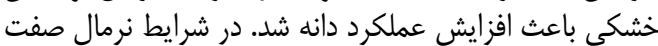

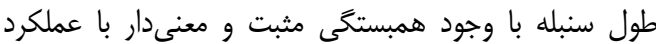

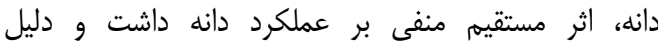

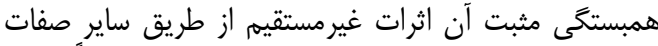

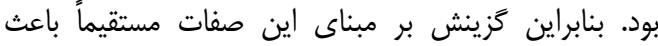

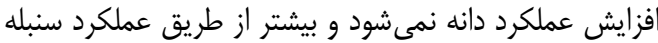

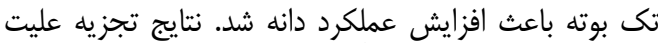

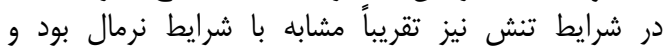

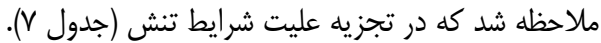

كه در شرايط نرمال صفات عملكرد سنبله تك بوته، تعداد دانه

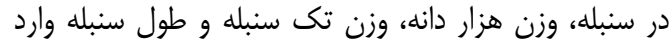

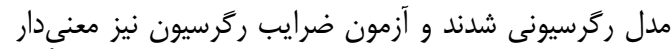

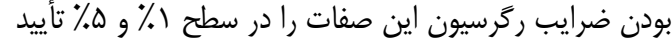

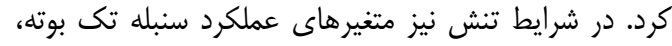

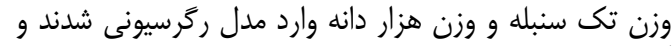

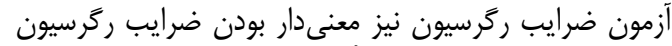

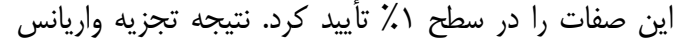

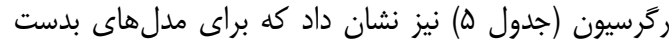

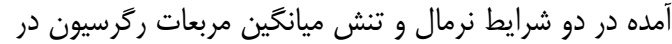

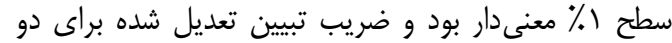

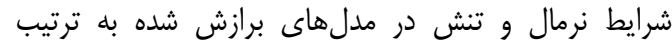

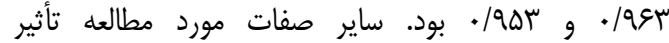
معنى دارى را در مدل رگرسيون نداشتند. با استفاده از تجزيه

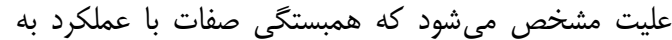

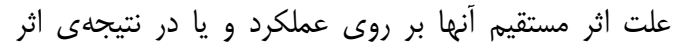

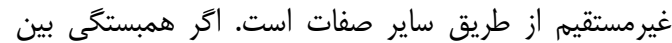

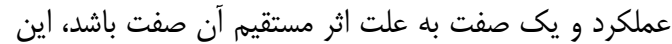
مطلب منعكس كنندهى يك رابطه واقعى بين آنها است و و لذا

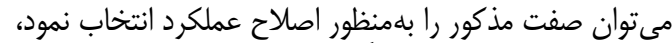

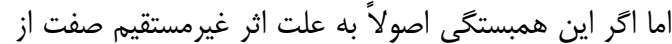

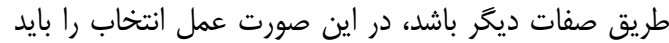

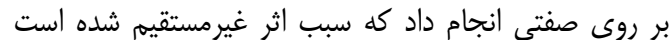

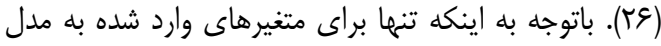

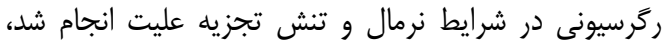

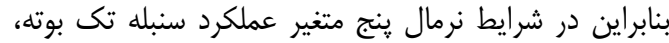

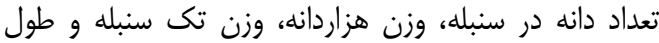

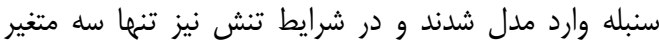

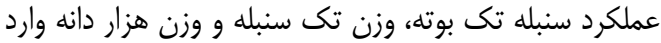

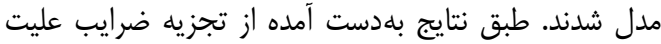

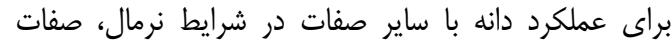

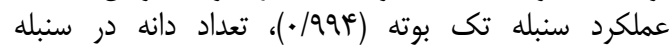

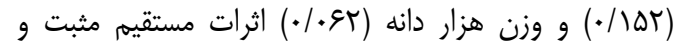

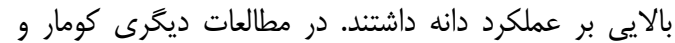

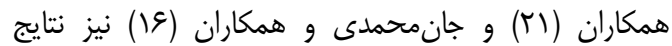

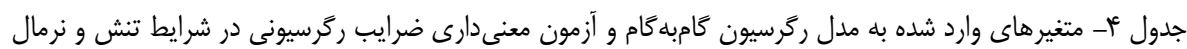
Table 4. The variables entered into the stepwise regression model and the significance test of regression coefficients

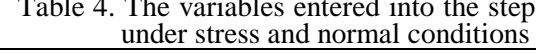

\begin{tabular}{|c|c|c|c|}
\hline \multicolumn{2}{|c|}{ شر ايط تنش } & \multicolumn{2}{|c|}{ شرايط نرمال } \\
\hline ضرايب ركَرسيون & متغير هاى وارد شده به مدل & ضرايب ركَر سيون & متغير هاى وارد شده به مدل \\
\hline$\cdot /$ AT n ns & ثابت ركر سيون & $-\cdot|\varnothing \Delta|^{\mathrm{ns}}$ & ثابت ركرسيون \\
\hline.$/ F \vee \% * *$ & عملكرد سنبله تك بوته & $\cdot / F Y$ KT & عملكرد سنبله تك بوته \\
\hline$-\varphi / \Lambda \Delta \uparrow * *$ & وزن تك سنبله & . Tr\&\&** & تعداد دانه در سنبله \\
\hline \multirow[t]{3}{*}{$\cdot / \cdot \mathrm{V} \mid * *$} & وزن هزار دانه & $\cdot / \cdot v) *$ & وزن هزار دانه \\
\hline & & $-\Upsilon / ৭ ९ 9 *$ & وزن تك سنبله \\
\hline & &.$- / M r 4 *$ & طول سنبله \\
\hline
\end{tabular}


جدول هـ- تجزيه واريانس رگرسيون گامبه گام و ضريب تبيين تعديل شده براى مدل برازش شده در شرايط تنش و نرمال Table 5. Variance analysis of stepwise regression and adjusted determination coefficient for fitted model under stress

\begin{tabular}{|c|c|c|c|c|}
\hline \multicolumn{2}{|c|}{ شرايط تنش } & \multicolumn{2}{|c|}{ شرايط نرمال } & \multirow{2}{*}{ منابع تغييرات } \\
\hline ميانگين مربعات & درجه آزادى & ميانگَين مربعات & درجه آزادى & \\
\hline$\Delta q / 9 \uparrow \wedge^{* * *}$ & $r$ & $V \& / \& q \mid * *$ & $\Delta$ & ركرسيون \\
\hline.$/ 19$ & fef & . & et & باقيمانده \\
\hline \multicolumn{2}{|c|}{ 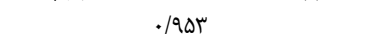 } & \multicolumn{2}{|c|}{ 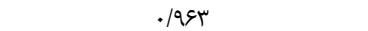 } & ضريب تبيين تعديل شده (R²) \\
\hline
\end{tabular}

جدول و- تجزيه ضرايب عليت اثرات مستقيه و غير مستقيم صفات ارزيابى شده بر روى عملكرد دانه تك بوته آزيلويس سيلندريكا در شرايط نرمال

Table 6. Path coefficients analysis of direct and indirect effects of evaluated traits on the grain yield of a single plant

\begin{tabular}{ccccccccc}
\multicolumn{2}{c}{ of Ae. cylindrica under normal conditions } \\
\hline
\end{tabular}

جدول V- تجزيه ضر ايب عليت اثرات مستقيم و غير مستقيم صفات ارزيابى شده بر روى عملكرد دانه تك بوته آزيلويس سيلندريكا در شرايط تنش خشكى

Table 7. Path coefficients analysis of direct and indirect effects of evaluated traits on the grain yield of a single plant

\begin{tabular}{|c|c|c|c|c|c|c|}
\hline \multirow{2}{*}{ صفات } & \multirow{2}{*}{ اثرات مستقيم } & \multicolumn{3}{|c|}{ اثرات غيرمستقيم } & \multirow{2}{*}{ غجموع اثرات } & \multirow{2}{*}{ كل } \\
\hline & & وزن ... إنه (g) & وزن تك سنبله (g) & عملكرد سنبله تك بوته (g) & & \\
\hline وزن ... (g) دانه (g) & $\cdot / \cdot{ }^{\prime}$ & --- & $-\cdot / \cdot \cdot 9$ & $\cdot / \cdot V r$ &.$/ .9 \mu$ &.$/$ MF \\
\hline وزن تك سنبله (g) & 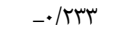 &..$\mu$ & --- & 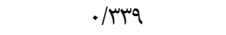 & . &.$/ 1.9$ \\
\hline عملكرد سنبله تك بوته (g) & $1 / \cdot r$ & .1 .94 & $-\cdot / \cdot \mathrm{W}$ & --- & $-\cdot|\cdot v|$ & $\cdot / 9 \Delta$. \\
\hline
\end{tabular}

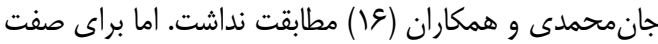

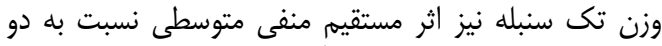

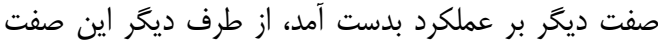

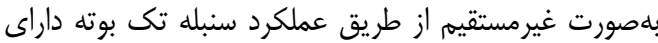

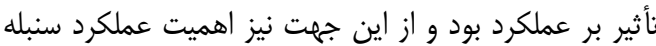

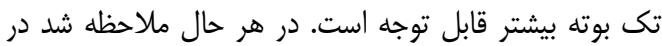

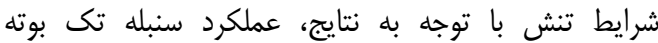

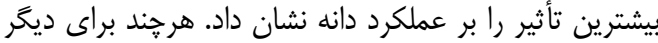

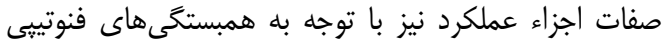

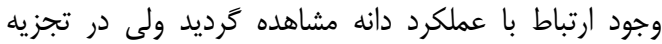

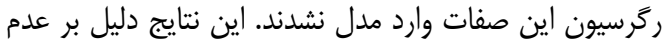

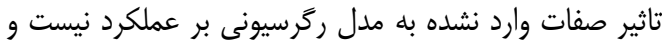

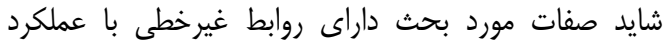

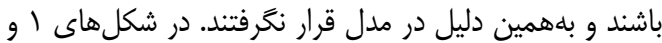

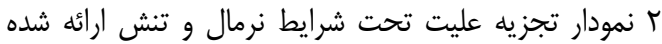

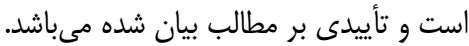

عملكرد سنبله تك بوته بيشترين اثر مستقيم را داشت إتاته

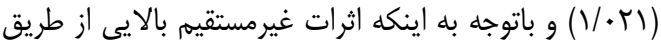

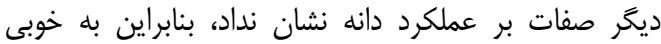

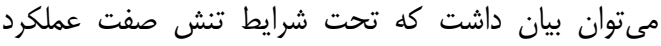

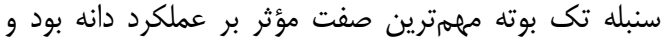

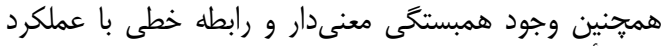

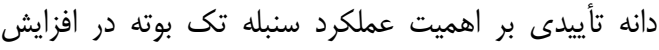

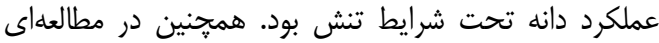

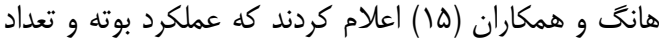

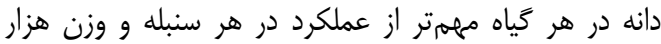

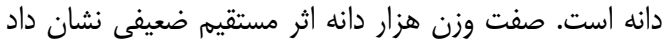

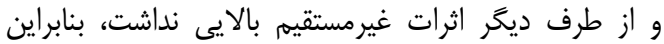

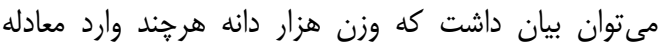

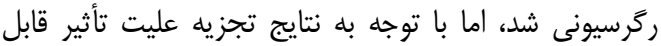

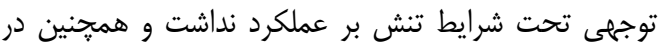
بخش همبستخى ها نيز رابطه معنى دارى نشى نشان نداد كه با نتايج 


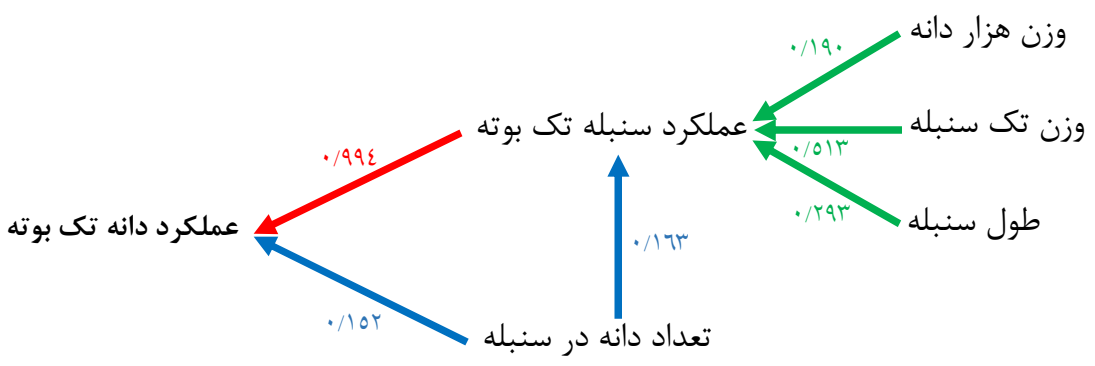

شكل ا- نمودار تجزيه عليت صفات مؤثر بر عملكرد دانه در شرايط نرمال با حذف اثرات ضعيف

Figure 1. The diagram of path analysis of effective traits on grain yield under normal conditions with remove of weak effects

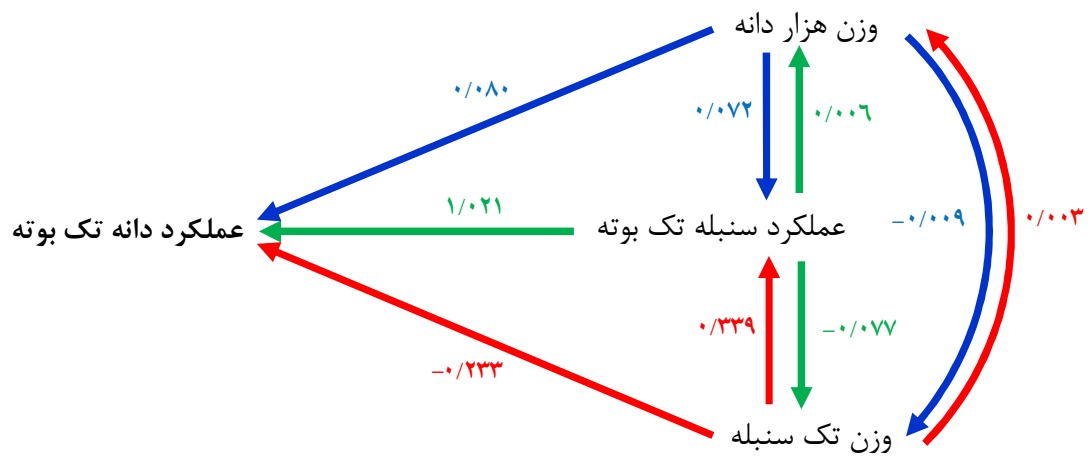

شكل r- نمودار تجزيه عليت صفات مؤثر بر عملكرد دانه در شرايط تنش خشكي

Figure 2. The diagram of path analysis of effective traits on grain yield under stress conditions

1. Aghaee, MJ., MR Naghavi, A R. Taleei, M. Omidi and J Mozafari. 2007. A study of chromosome homology between three Iranian Aegilops species with D genome and bread wheat (T. aestivum). Iranian Journal of Rangelands and Forests Plant Breeding and Genetic Research, 15: 95-112 (In Persian).

2. Akram, Z., S. Ajmal and M. Munir. 2008. Estimation of correlation coefficient among some yield parameters of wheat under rainfed conditions. Pakistan Journal of Botany, 40: 1777-1781.

3. Alishah, A. and M. Omidi. 2008. Laboratory methods in plant cytogenetics, Tehran University Press, $188 \mathrm{pp}$ (In Persian).

4. Amiri, S., S. Nour Mohammadi, A.A. Jafari and R. Chougan. 2009. Correlation, regression and path analysis for grain yield and yield components on early maturing hybrids of grain corn. Journal of Plant Production, 16: 99-112.

5. Anwar, J., M.A. Ali, M. Hussain, W. Sabir, M.A. Khan, M. Zulkiffal and M. Abdullah. 2009. Assessment of yield criteria in bread wheat through correlation and path analysis. Journal of Animal and Plant Sciences, 19: 185-188.

6. Ata, A., B. Yousaf, A.S. Khan, G. Mahboob Subhani, H.M. Asadullah and A. Yousaf. 2014. Correlation and path coefficient analysis for important plant attributes of spring wheat under normal and drought stress conditions. Journal of Biology, Agriculture and Healthcare, 4: 23-28.

7. Bluman, P. and L.A. Hunt. 1988. Relationships among tillering, spike number and grain yield in winter wheat in Ontario. Canadian Journal of Plant Science, 68: 583-596.

8. Chowdhry, M.A., M. Ali, G.M. Subhani and I. Khaliq. 2000. Path coefficient analysis for water use efficiency, evapo-transpiration efficiency and some yield related traits in wheat at different micro environments. Environmental Ecology, 9: 906-10.

9. Doffing, S.M. and C.W. Knight. 1992. Alternative model for path analysis of small grain yield. Crop Science, 32: 487- 489.

10. Farshadfar, E. 1998. Application of biometrical genetics in plant breeding. vol. I, Razi University Press, $130 \mathrm{pp}$ (In Persian).

11. Gandhi, H.T., M.I.Vales, C.J.W. Watson, C. Mallory-Smith, N. Mori, M. Rehman, R.S. Zemetra and O. Riera-Lizarazu. 2005. Chloroplast and nuclear microsatellite analysis of Aegilops cylindrica Theoretical and Applied Genetics, 111: 561-572. 
12. Gohari, A.M., N. Sedaghat, M. Javan nikkhah and R. Saberi-Riseh. 2007. Mycoflora of wheat grains in the main production area in Kerman province, Iran. International Journal of Agriculture and Biology, 9: 635-637 (In Persian).

13. Guertin, W.H. and J.P. Bailey.1982. Introduction to modern Factor analysis. Edwards Brothers. Inc., Michigan, $472 \mathrm{pp}$.

14. Hosseinpour, T., R. Mamghani, A.A. Siadat and M. Bahari. 2003. Path analysisof agronomic traitsforgrain andstraw yield of wheat genotypesunder limitedirrigation condition. Scientific Journal of Agriculture, 26: 105-120 (In Persian).

15. Huang, Z., Z. Meixue and H. Yousheng. 1989. Analysis of genetic advance, genetic correlation and path coefficient for yield characters in wheat. Jiangsu Journal of Agricultural Sciences (China), 5: 1823.

16. Janmohammadi, M., N. Sabaghnia and M. Nouraein. 2014. Path analysis of grain yield and yield components and some agronomic traits in bread wheat. Acta Universitatis Agriculturae et Silviculturae Mendelianae Brunensis, 62: 945-952.

17. Kashif, M. and I. Khaliq. 2004. Heritability, correlation and path coefficient analysis for some metric traits in wheat. International Journal of Agriculture and Biology, 1: 138-142.

18. Keim, D.L. and W.E. Kronstand. 1981. Drought responses of winter wheat cultivars grown under field stress conditions. Crop Science, 21: 11-14.

19. Khan, N. and F.N. Naqvi. 2012. Correlation and path coefficient analysis in wheat genotypes under irrigated and non-irrigated conditions. Asian Journal of Agricultural Sciences, 4: 346-351.

20. Khodadadi, M., H. Dehghani and M.H. Fotokian. 2011. Study of heritability, path and factor analysis in winter wheat (Triticum aestivum L.) genotypes. Journal of Agronomy Sciences, 4: 67-78 (In Persian).

21. Kumar, R., B. Bhushan, R. Pal and S.S.Gaurav. 2014. Correlation and path coefficient analysis for quantitative traits in wheat (Triticum aestivum L.) under normal condition. Annals of Agri Bio Research, 19: 447-450.

22. Li, W., Z.H. Yan, Y.M. Wei, X.J. Lan and Y.L. Zheng. 2006. Evaluation of genotype $\times$ environment interactions in Chinese spring wheat by the AMMI model, correlation and path analysis. Journal of Agronomy and Crop Science, 192: 221-227.

23. Moghaddam, M., B. Ehdaie and J.G. Waines. 1997. Genetic variation and interrelationships among agronomic traits in landraces of bread wheat from southwestern Iran. Euphytica, 95: 361-369.

24. Mollasadeghi, V., A.A. Imani, R. Shahryari and M. Khayatnezhad. 2011. Correlation and path analysis of morphological traits in different wheat genotypes under end drought stress condition. Middle-East Journal of Scientific Research, 7: 221-224.

25. Mondal, A.B., D.P. Sadhu and K.K. Sarkar. 1997. Correlation and path analysis in bread wheat. Environmental Ecology, 15: 537-539.

26. Nasri, R., F. Paknejad, M. Sadeghi Shoa, S. Ghorbani and Z. Fatemi. 2013. Correlation and path analysis of drought stress on yield and yield components of barley (Hordeum vulgare) in Karaj region. Iranian Journal of Agronomy and Plant Breeding, 8: 155-165 (In Persian).

27. Pour Aboughadareh, A., S.S. Alavikia, M. Moghaddam, A.A. Mehrabi and M.A. Mazinani. 2016. Diversity of agro morphological traits in populations of einkorn wheat (Triticum boeoticum and Triticum urartu) under normal and water deficit stress conditions. Journal of Crop Breeding, 8: 37-46 (In Persian).

28. Pourmoradi, S. and H. Mirzaie-Nodoushan. 2011. Path analysis of morphological traits and forage yield on several populations of Lolium species. Iranian Journal of Rangelands and Forests Plant Breeding and Genetic Research, 18: 294-304 (In Persian).

29. Richards, R.A. 1996. Defining selection criteria improve yield under drought. Plant Growth Regulation, 20: 157-166.

30. Saberi, M.H., E. Arazmjoo and A. Amini. 2016. Assessment of Diversity and Identifying of Effective Traits on Grain Yield of bread wheat Promised Lines under Salt Stress Conditions. Journal of Crop Breeding, 8: 31-40 (In Persian).

31. Saleem, U., I. Khaliq, M. Tariq and M. Rafique. 2006. Phenotypic and genotypic correlation coefficients between yield and yield components in wheat. Journal of Agricultural Research, 44: 1-8.

32. Schneider, A., I. Molnar and M. Molnar-Lang. 2008. Utilization of Aegilops (goatgrass) species to widen the genetic diversity of cultivated wheat. Euphytica, 163: 1-19.

33. Shahid Masood, M., A. Javaid, M. Ashiq Rabbani and R. Anwar. 2005. Phenotypic diversity and trait association in bread wheat (Triticum aestivum L.) Landraces from Baluchistan, Pakistan. Pakistan Journal of Botany, 37: 949-957.

34. Shahid, M., M. Fida and M. Tahir. 2002. Path coefficient analysis in wheat. Sarhad Journal of Agriculture, 18: 383-388.

35. Shanahan, J.F., K.J. Donnelly, D.H. Smith and D.E. Smika. 1985. Shoot developmental properties associated with grain yield in Winter wheat. Crop Science, 25: 770-775.

36. Topal, A., C. Aydin, N. Akgün and M. Babaoglu. 2004. Diallel cross analysis in durum wheat (Triticum durum Desf.): identification of best parents for some kernel physical features. Field Crops Research, 87: 1-12.

37. Uddin, M.J., B. Mitra, M.A.Z. Chowdhry and B. Mitra. 1997. Genetic parameters, correlation path coefficient and selection indices in wheat. Bangladesh Journal of Science and Industry Research, 32: 528-538.

38. Zakizadeh, M., M. Esmaeilzadeh Moghaddam and D. Kahrizi. 2010. Study on genetic variation and relationship between plant characteristics and grain yield in long spike bread wheat (Triticum aestivum L.) genotypesusing multivariate analysis. Iranian Journal of Crop Science, 12: 18-30. 


\title{
Study of Correlation and Path Coefficient Analysis of Agronomic Traits and Grain Yield for Aegilops cylindrica Accessions under Non-Stress and Drought Stress Conditions in Ilam
}

\author{
Afsaneh Noori ${ }^{1}$, Ali Ashraf Mehrabi ${ }^{2}$ and Hoshmand Safari ${ }^{3}$ \\ 1- M.Sc. Student, Faculty of Agriculture and Natural Resources, Ilam University \\ 2- Associated Professor, Faculty of Agriculture, Ilam University (Corresponding author: a.mehrabi@ilam.ac.ir) \\ 3- Faculty Member of Research Department of Forests and Rangelands,Kermanshah Agricultural and Natural \\ Resources Research and EducationCenter, Agricultural Research, Education and Extension Organization \\ (AREEO), Kermanshah, Iran \\ Received: November 27, $2015 \quad$ Accepted: July 4, 2016
}

\begin{abstract}
In order to study the relationships between grain yield and its components of Aegilops cylindrica, 48 accessions of this plant species was studied in an experimental augmented design based on randomized complete block designwith4 replications under normal and drought stress conditions in the Agricultural Research Station of Ilam University. The results of correlation analysis between the traits in both normal and stress conditions suggested that spike yield per plant, biological yield per plant and straw weight per plant had the highest correlation with the grain yield per plant. The results of regression analysis showed that the spike yield per plant, number of grains per spike, 1000 grain weight, single spike weight and spike length in normal conditions and spike yield per plant, single spike weight and 1000 grain weight in stress condition were entered into regression model. 96.3 and 95.3 percent of the total of variance of the regression was expressed in normal and stress conditions, respectively. Also, path analysis confirmed the effects of these traits on grain yield. In both irrigated and drought conditions, spike yield per plant had the greatest positive direct effect on grain yield. The results showed that in breeding programs aimed at increasing grain yield in populations of Aegilops cylindrica, selection is better to be done through the more spike yield in both normal and drought conditions.
\end{abstract}

Keywords: Aegilops cylindrica, Correlation analysis, Drought stress, Grain yield, Path Coefficient analysis 